\title{
Mobile Network Energy Efficiency Optimization in MIMO Multi-Cell Systems
}

\author{
Yang Yang and Dario Sabella \\ Intel Deutschland GmbH, Neubiberg 85579, Germany. \\ Emails: yang1.yang@intel.com, dario.sabella@intel.com
}

\begin{abstract}
In this paper, we consider the energy efficiency maximization problem in downlink multi-input multi-output (MIMO) multi-cell systems, where all users suffer from intercell interference. To solve this optimization problem with a nonconcave objective function and a complex-valued matrix variable, we extend the recently developed successive pseudoconvex approximation framework and propose a novel iterative algorithm that has the following advantages: 1) fast convergence as the structure of the original optimization problem is preserved as much as possible in the approximate problem solved in each iteration, 2) easy implementation as each approximate problem is natural for parallel computation and its solution has a closed-form expression, and 3) guaranteed convergence to a stationary point. The advantages of the proposed algorithm are also illustrated numerically in terms of energy efficiency gains from mobile network infrastructure perspective.
\end{abstract}

Index Terms-Energy Efficiency, Interference-limited System, MIMO, Nonconvex Optimization, Pseudoconvex optimization

\section{INTRODUCTION}

In the era of $5 \mathrm{G}$ and Internet of Things by 2020, the number of connected devices is predicted to reach 50 billions. On one hand, the data rate should be 1000x faster to serve these devices simultaneously. On the other hand, the significant increase in the data rate is expected to be achieved at the same or even a lower level of energy consumption. Therefore the socalled energy efficiency (EE) is a key performance indicator that should be considered in the design of efficient transmission schemes enhancing the spectral and energy efficiency.

In this paper, we study the EE maximization problem in a downlink multi-input multi-output (MIMO) multi-cell system, where the users are sharing the same frequency resources and suffer from inter-cell interference. It is well known that sum rate maximization in such an interference-limited system is a nonconvex optimization problem and NP-hard [1]. The EE maximization problem in an interference-limited system is even more challenging because the $\mathrm{EE}$ is a fractional function where the nonconcave sum rate function is in the numerator and the consumed energy is an additional variable in the denominator [2].

In state-of-the-art studies, the EE maximization problem in interference-limited systems has received considerable attention, see [2, 3, 4, 5, 6, 7] for some recent examples and the references therein. An iterative algorithm was proposed in [2] and [3] to maximize the EE in a single-input singleoutput (SISO) system and a multi-input single-output (MISO) system, respectively, but they are not applicable for a MIMO system. They also suffer from a high complexity, because, although the optimization problem solved in each iteration is convex, it does not have structures that can be exploited to enable, e.g., parallel computation, and it can only be solved by general purpose optimization solvers and this may incur a large latency in the decision making process. The sequential pricing algorithm proposed in [5] is a variant of the wellknown block coordinate descent algorithm. Although it can be extended to a MIMO system, the optimization problem solved in each iteration does not exhibit any convexity, making the iterative algorithm not suitable for practical implementation. A general framework for EE optimization based on monotonic programming is proposed in a recent paper [7], but it is not applicable for MIMO systems where the optimization variables are complex-valued matrices.

In this paper, we extend the recently developed successive pseudoconvex approximation (SPCA) framework proposed in [6] to solve the EE maximization problems in MIMO interference-limited systems. In each iteration, an approximate problem is solved, and the approximate function only needs to be pseudoconvex, a weak form of convexity. This weak assumption makes it possible to preserve as much structure available in the original EE function as possible, e.g., the partial concavity in the numerator function and the division operator. Besides this, the proposed approximate problem is natural for parallel computation, as the approximate problem can be decomposed into many independent subproblems that can be solved in parallel and each subproblem has a closedform solution. Therefore, the proposed algorithm presents a fast convergence behavior and enjoys an easy implementation.

\section{PRoblem Model}

We consider a downlink MIMO multi-cell system. We assume the number of cells is $K$, and each cell is serving one user. This assumption is considered realistic especially in practical dense urban scenarios, where instantaneously the number of active users connected to a single BS at a specific frequency is low (most of the time only one single user per small cell). The number of transmit antennas at the base station (BS) of cell $k$ is $M_{T, k}$, and the number of receive antennas of user $k$ served by cell $k$ is $M_{R, k}$. We denote $\mathbf{H}_{k k}$ as the channel coefficient from BS $k$ to user $k$, and $\mathbf{H}_{k j}$ as the channel coefficient from BS $j$ to user $k$. We assume that all $K$ users are active and the inter-cell interference is treated as noise, so the downlink transmission rate towards the $k$-th user 


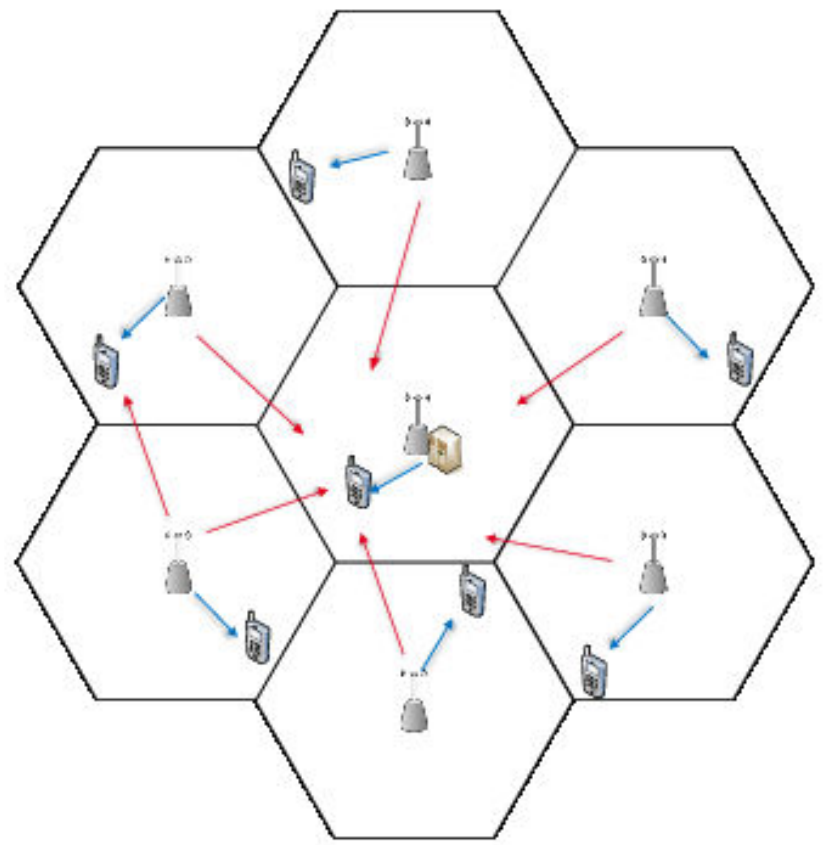

Figure 1. System topology with 1-tiered small cell interferers and central processing unit in CRAN

is:

$$
r_{k}\left(\mathbf{Q}_{k}, \mathbf{Q}_{-k}\right)=\log \operatorname{det}\left(\mathbf{I}+\mathbf{R}_{k}\left(\mathbf{Q}_{-k}\right)^{-1} \mathbf{H}_{k k} \mathbf{Q}_{k} \mathbf{H}_{k k}^{H}\right),
$$

where $\mathbf{Q}_{k} \triangleq \mathbb{E}\left[\mathbf{x}_{k} \mathbf{x}_{k}^{H}\right]$ (with $\mathbf{x}_{k}$ being the transmitted symbol) is BS $k$ 's transmit covariance matrix, $\mathbf{Q}_{-k}$ is a compact notation denoting all transmit covariance matrices except $\mathbf{Q}_{k}$ : $\mathbf{Q}_{-k}=\left(\mathbf{Q}_{j}\right)_{j \neq k}$, and $\mathbf{R}_{k}\left(\mathbf{Q}_{-k}\right) \triangleq \sigma_{k}^{2} \mathbf{I}+\sum_{j \neq k} \mathbf{H}_{k j} \mathbf{Q}_{j} \mathbf{H}_{k j}^{H}$ is the noise plus interference covariance matrix experienced by user $k$.

The power consumption at BS $k$ can be approximated by the following equation [8, Section 4.3]:

$$
P_{0, k}+\rho_{k} \operatorname{tr}\left(\mathbf{Q}_{k}\right),
$$

where $P_{0, k}$ is the power consumption at the zero RF output power (i.e., $\mathbf{Q}_{k}=\mathbf{0}$ ), and $\rho_{k}$ is the slope of the load dependent power consumption. The concrete values of $P_{0, k}$ and $\rho_{k}$ depend on the types of the cell, e.g., macro cell, remote radio head, and micro cell. Interested readers can refer to $[8$, Table 8].

The EE maximization problem at the network level is then formulated as the ratio between the sum transmission rate in the multi-cell system and the total consumed power:

$$
\begin{array}{ll}
\underset{\mathbf{Q}}{\operatorname{maximize}} & f(\mathbf{Q}) \triangleq \frac{\sum_{k=1}^{K} r_{k}\left(\mathbf{Q}_{k}, \mathbf{Q}_{-k}\right)}{\sum_{k=1}^{K}\left(P_{0, k}+\rho_{k} \operatorname{tr}\left(\mathbf{Q}_{k}\right)\right)} \\
\text { subject to } & \mathbf{Q}_{k} \succeq \mathbf{0}, \operatorname{tr}\left(\mathbf{Q}_{k}\right) \leq P_{k}, \forall k,
\end{array}
$$

where $P_{k}$ is BS $k$ 's (predefined) sum transmission power budget and the optimization variable is the (complex-valued) transmit covariance matrices $\mathbf{Q} \triangleq\left(\mathbf{Q}_{k}\right)_{k=1}^{K}$. Note that the objective function of (1) has a unit of bits/Joule and it specifies the EE of the entire cluster depicted in Figure 1).

\section{The Proposed Iterative Algorithm For SUM-RATE-SUM-POWER RATIO MAXIMIZATION}

Problems (1) is known to be nonconvex and NP-hard, and we aim at developing an algorithm that can efficiently find a stationary point. Note that a stationary point is a point that satisfies the necessary optimality conditions of problems (1), and it is the classical goal of algorithmic design for nonconvex problems [9].

To design an iterative algorithm for problem (1) that enjoys a low complexity but at the same time a fast convergence behavior, we need on one hand to overcome the nonconvexity in the objective function and, on the other hand, to preserve the original problem's structure as much as possible. Towards this end, we propose an iterative algorithm based on the successive pseudoconvex approximation framework developed in [6].

To start with, we introduce the definition of pseudoconvex functions: a function $f(\mathbf{x})$ is said to be pseudoconvex if [10]

$$
f(\mathbf{y})<f(\mathbf{x}) \Longrightarrow(\mathbf{y}-\mathbf{x})^{T} \nabla f(\mathbf{x})<0 .
$$

In other words, $f(\mathbf{y})<f(\mathbf{x})$ implies $\mathbf{y}-\mathbf{x}$ is a descent direction of $f(\mathbf{x})$ [9]. A function $f(\mathbf{x})$ is pseudoconcave if $-f(\mathbf{x})$ is pseudoconvex. We remark that the (strong) convexity of a function implies that the function is pseudoconvex, which in turn implies that the function is quasi-convex.

The proposed iterative algorithm for problem (1) consists of solving a sequence of successively refined approximate problems. In iteration $t$, the approximate problem defined around the point $\mathbf{Q}^{t}$ consists of maximizing an approximatie function, denoted as $\tilde{f}\left(\mathbf{Q} ; \mathbf{Q}^{t}\right)$, under the same constraints as (1). The lack of concavity in the objective function should be properly compensated so that the approximate problems are much easier to solve than the original problem (1).

The numerator functions $\left\{r_{k}(\mathbf{Q})\right\}$ are not concave in $\mathbf{Q}$, and thus the objective function $f(\mathbf{Q})$ is not even pseudoconcave, a weaker form of convexity that is recognized to play an essential role in many optimization problems $[6,10]$. Meanwhile, the function $r_{k}(\mathbf{Q})$ is concave in $\mathbf{Q}_{k}$, and exploiting this partial concavity may notably accelerate the convergence [11]. Therefore, we approximate the numerator function $\sum_{j=1}^{K} r_{j}(\mathbf{Q})$ with respect to $\mathbf{Q}_{k}$ at the point $\mathbf{Q}^{t}$ by a function denoted as $\tilde{r}_{k}\left(\mathbf{Q}_{k} ; \mathbf{Q}^{t}\right)$, which is obtained by fixing the other variables $\mathbf{Q}_{-k}$ in $r_{k}\left(\mathbf{Q}_{k}, \mathbf{Q}_{-k}\right)$ and linearizing only the functions $\left\{r_{j}(\mathbf{Q})\right\}_{j \neq k}$ that are not concave in $\mathbf{Q}_{k}$ :

$\tilde{r}_{k}\left(\mathbf{Q}_{k} ; \mathbf{Q}^{t}\right) \triangleq r_{k}\left(\mathbf{Q}_{k}, \mathbf{Q}_{-k}^{t}\right)+\sum_{j \neq k}\left(\mathbf{Q}_{k}-\mathbf{Q}_{k}^{t}\right) \bullet \nabla_{\mathbf{Q}_{k}^{*}} r_{j}\left(\mathbf{Q}^{t}\right)$,

where $\mathbf{X} \bullet \mathbf{Y} \triangleq \Re\left(\operatorname{tr}\left(\mathbf{X}^{H} \mathbf{Y}\right)\right)$ and $\nabla_{\mathbf{Q}_{k}^{*}} r_{j}(\mathbf{Q})$ is the Jacobian matrix of $r_{j}(\mathbf{Q})$ with respect to $\mathbf{Q}_{k}^{*}$ (the conjugate of $\mathbf{Q}_{k}$ ). Since $\tilde{r}_{k}\left(\mathbf{Q}_{k} ; \mathbf{Q}^{t}\right)$ is concave in $\mathbf{Q}_{k}, \sum_{k=1}^{K} \tilde{r}_{k}\left(\mathbf{Q}_{k} ; \mathbf{Q}^{t}\right)$ is concave in $\mathbf{Q}$. This paves the way to define the following approximate function of the original objective function $f(\mathbf{Q})$ at point $\mathbf{Q}^{t}$, denoted as $\tilde{f}\left(\mathbf{Q} ; \mathbf{Q}^{t}\right)$ :

$$
\tilde{f}\left(\mathbf{Q} ; \mathbf{Q}^{t}\right) \triangleq \frac{\sum_{k=1}^{K} \tilde{r}_{k}\left(\mathbf{Q}_{k} ; \mathbf{Q}^{t}\right)}{\sum_{k=1}^{K}\left(P_{0, k}+\rho_{k} \operatorname{tr}\left(\mathbf{Q}_{k}\right)\right)} .
$$

The approximate function $\tilde{f}\left(\mathbf{Q} ; \mathbf{Q}^{t}\right)$ has some important properties as we outline. 
Firstly, the approximate function $\tilde{f}\left(\mathbf{Q} ; \mathbf{Q}^{t}\right)$ is still nonconcave, but it is a fractional function between a nonnegative concave function and a positive linear function, which is thus pseudoconcave [6].

Secondly, the approximate function $f\left(\mathbf{Q} ; \mathbf{Q}^{t}\right)$ is differentiable and its gradient is the same as that of the original function $f(\mathbf{Q})$ at the point $\mathbf{Q}^{t}$ where the approximate function $f\left(\mathbf{Q} ; \mathbf{Q}^{t}\right)$ is defined. To see this, we remark that $\left.\nabla_{\mathbf{Q}_{k}^{*}} \tilde{r}_{j}\left(\mathbf{Q}_{j} ; \mathbf{Q}^{t}\right)\right|_{\mathbf{Q}=\mathbf{Q}^{t}}=\mathbf{0}$ if $j \neq k$, and

$$
\begin{aligned}
\left.\nabla_{\mathbf{Q}_{k}^{*}} \tilde{r}_{k}\left(\mathbf{Q}_{k} ; \mathbf{Q}^{t}\right)\right|_{\mathbf{Q}=\mathbf{Q}^{t}} & =\left.\nabla_{\mathbf{Q}_{k}^{*}}\left(\sum_{j=1}^{K} r_{j}(\mathbf{Q})\right)\right|_{\mathbf{Q}=\mathbf{Q}^{t}}, \\
\tilde{r}\left(\mathbf{Q}^{t} ; \mathbf{Q}^{t}\right) & =r_{k}\left(\mathbf{Q}^{t}\right)
\end{aligned}
$$

Based on the observations in (4)-(5), it can be verified that

$$
\begin{aligned}
& \left.\nabla_{\mathbf{Q}_{j}^{\star}} \tilde{f}\left(\mathbf{Q} ; \mathbf{Q}^{t}\right)\right|_{\mathbf{Q}=\mathbf{Q}^{t}} \\
= & \frac{\nabla_{\mathbf{Q}_{j}^{*}}\left(\sum_{k=1}^{K} \tilde{r}_{k}\left(\mathbf{Q}_{k}^{t} ; \mathbf{Q}^{t}\right)\right) \mid}{\sum_{k=1}^{K}\left(P_{0, k}+\rho_{k} \operatorname{tr}\left(\mathbf{Q}_{k}^{t}\right)\right)}-\frac{\left(\sum_{k=1}^{K} \tilde{r}_{k}\left(\mathbf{Q}_{k}^{t} ; \mathbf{Q}^{t}\right)\right) \mathbf{I}}{\left(\sum_{k=1}^{K}\left(P_{0, k}+\rho_{k} \operatorname{tr}\left(\mathbf{Q}_{k}^{t}\right)\right)\right)^{2}} \\
= & \frac{\nabla_{\mathbf{Q}_{j}^{*}}\left(\sum_{k=1}^{K} r_{k}\left(\mathbf{Q}^{t}\right)\right)}{\sum_{k=1}^{K}\left(P_{0, k}+\rho_{k} \operatorname{tr}\left(\mathbf{Q}_{k}\right)\right)}-\frac{\left(\sum_{k=1}^{K} r_{k}\left(\mathbf{Q}^{t}\right)\right) \mathbf{I}}{\left(\sum_{k=1}^{K}\left(P_{0, k}+\rho_{k} \operatorname{tr}\left(\mathbf{Q}_{k}\right)\right)\right)^{2}} \\
= & \nabla_{\mathbf{Q}_{j}^{*}}\left(\frac{\sum_{k=1}^{K} r_{k}\left(\mathbf{Q}^{t}\right)}{\sum_{k=1}^{K}\left(P_{0, k}+\rho_{k} \operatorname{tr}\left(\mathbf{Q}_{k}^{t}\right)\right)}\right)=\left.\nabla_{\mathbf{Q}_{j}^{*}} f(\mathbf{Q})\right|_{\mathbf{Q}=\mathbf{Q}^{t}} .
\end{aligned}
$$

These properties coincide with those given in [6] and have been shown to play an essential role in establishing the convergence properties.

At iteration $t$ of the proposed algorithm, the approximate problem defined at the point $\mathbf{Q}^{t}$ is to maximize the approximate function $\tilde{f}\left(\mathbf{Q} ; \mathbf{Q}^{t}\right)$ defined in (3) subject to the same constraints as in the original problem (1):

$$
\begin{array}{ll}
\underset{\mathbf{Q}}{\operatorname{maximize}} & \tilde{f}\left(\mathbf{Q} ; \mathbf{Q}^{t}\right) \\
\text { subject to } & \mathbf{Q}_{k} \succeq \mathbf{0}, \operatorname{tr}\left(\mathbf{Q}_{k}\right) \leq P_{k}, k=1, \ldots, K .
\end{array}
$$

We denote as $\mathbb{B} \mathbf{Q}^{t}$ the (unique) solution of problem (7a):

$$
\mathbb{B} \mathbf{Q}^{t} \triangleq \underset{\left(\mathbf{Q}_{k} \succeq \mathbf{0}, \operatorname{tr}\left(\mathbf{Q}_{k}\right) \leq P_{k}\right)_{k=1}^{K}}{\arg \max } \tilde{f}\left(\mathbf{Q} ; \mathbf{Q}^{t}\right) .
$$

Due to the pseudoconcavity, differentiability and equal gradient at $\mathbf{Q}^{t}$ as discussed above, the approximate function $\tilde{f}\left(\mathbf{Q} ; \mathbf{Q}^{t}\right)$ defined in (3) satisfies the assumptions specified in [6, A1-A3]. As a result, $\mathbb{B} \mathbf{Q}^{t}-\mathbf{Q}^{t}$ is an ascent direction of the original objective function $f(\mathbf{Q})$ at $\mathbf{Q}=\mathbf{Q}^{t}$, unless $\mathbf{Q}^{t}$ is already a stationary point of problem (1), as stated in the following proposition.

Proposition 1 (Stationary point and ascent direction). A point $\mathbf{X}$ is a stationary point of (1) if and only if $\mathbf{X}=\mathbb{B} \mathbf{X}$. If $\mathbf{X}$ is not a stationary point of (1), then $\mathbb{B} \mathbf{X}-\mathbf{X}$ is an ascent direction of $r(\mathbf{Q})$ in the sense that

$$
(\mathbb{B} \mathbf{X}-\mathbf{X}) \bullet \nabla f(\mathbf{X})>0 .
$$

Proof: The proof follows from the same line of analysis as that of [6, Proposition 1].

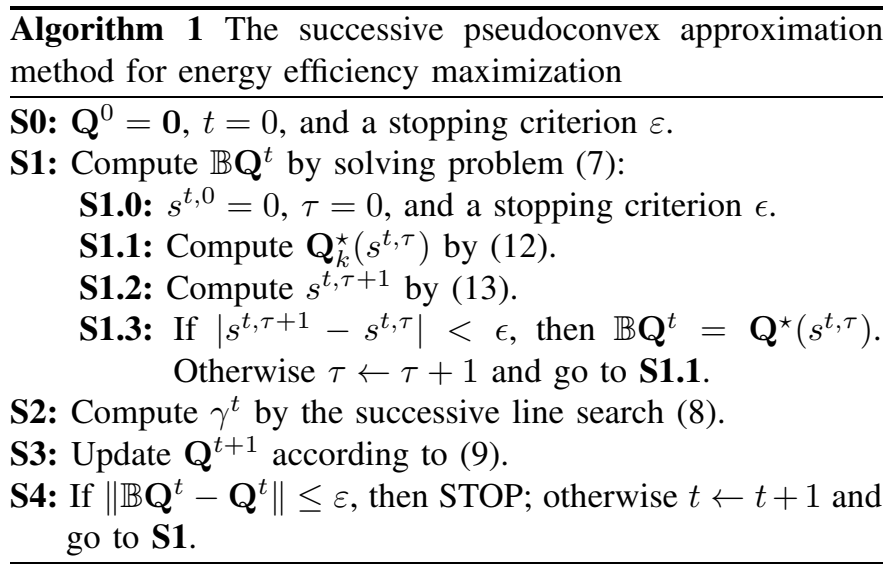

Since $\mathbb{B} \mathbf{Q}^{t}-\mathbf{Q}^{t}$ is an ascent direction of $f(\mathbf{Q})$ at $\mathbf{Q}=\mathbf{Q}^{t}$ according to Proposition 1, there exists a scalar $\gamma^{t} \in(0,1]$ such that $f\left(\mathbf{Q}^{t}+\gamma^{t}\left(\mathbb{B} \mathbf{Q}^{t}-\mathbf{Q}^{t}\right)\right)>f\left(\mathbf{Q}^{t}\right)$. In practice, the stepsize $\gamma^{t}$ is usually obtained by the so-called successive line search. That is, given two scalars $0<\alpha<1$ and $0<\beta<1$, $\gamma^{t}$ is set to be $\gamma^{t}=\beta^{m_{t}}$, where $m_{t}$ is the smallest nonnegative integer $m$ satisfying the following inequality:

$f\left(\mathbf{Q}^{t}+\beta^{m}\left(\mathbb{B} \mathbf{Q}^{t}-\mathbf{Q}^{t}\right)\right) \geq f\left(\mathbf{Q}^{t}\right)+\alpha \beta^{m} \nabla f\left(\mathbf{Q}^{t}\right) \bullet\left(\mathbb{B} \mathbf{Q}^{t}-\mathbf{Q}^{t}\right)$.

Note that the successive line search is carried out over the original objective function $f(\mathbf{Q})$ defined in (1).

After the stepsize $\gamma^{t}$ is found, the variable $\mathbf{Q}$ is updated as follows:

$$
\mathbf{Q}^{t+1}=\mathbf{Q}^{t}+\gamma^{t}\left(\mathbb{B} \mathbf{Q}^{t}-\mathbf{Q}^{t}\right)
$$

The resulting sequence $\left\{f\left(\mathbf{Q}^{t}\right)\right\}_{t}$ is monotonically increasing:

$$
\begin{aligned}
f\left(\mathbf{Q}^{t+1}\right) & =f\left(\mathbf{Q}^{t}+\beta^{m_{t}}\left(\mathbb{B} \mathbf{Q}^{t}-\mathbf{Q}^{t}\right)\right) \\
& \geq f\left(\mathbf{Q}^{t}\right)+\alpha \beta^{m_{t}} \nabla f\left(\mathbf{Q}^{t}\right) \bullet\left(\mathbb{B} \mathbf{Q}^{t}-\mathbf{Q}^{t}\right) \\
& \geq f\left(\mathbf{Q}^{t}\right), \forall t,
\end{aligned}
$$

where (10a) and (10b) come from the definition of the successive line search (8), and (10c) comes from Proposition 1.

The proposed algorithm is formally summarized in Algorithm 1 and its convergence properties are given in the following theorem.

Theorem 2 (Convergence to a stationary point). The sequence $\left\{\mathbf{Q}^{t}\right\}$ generated by Algorithm 1 has a limit point, and every limit point is a stationary point of problem (1).

Proof: The constraint set of problem (1), namely, $\left\{\left(\mathbf{Q}_{k}\right)_{k=1}^{K}: \mathbf{Q}_{k} \succeq \mathbf{0}, \operatorname{tr}\left(\mathbf{Q}_{k}\right) \leq P_{k}\right\}$, is nonempty and bounded. The sequence $\left\{\mathbf{Q}^{t}\right\}_{t}$ is thus bounded and has a limit point. Then the latter statement can be proved following the same line of analysis as [6] and thus not duplicated here.

In Step 1 of Algorithm 1, a constrained pseudoconvex optimization problem, namely, problem (7), must be solved. Since the optimal point $\mathbb{B} \mathbf{Q}^{t}$ does not have a closed-form expression, we apply the Dinkelbach's algorithm to solve problem (7) iteratively: at iteration $\tau$ of Dinkelbach's algorithm, the following problem is solved for a given and fixed $s^{t, \tau}\left(s^{t, 0}\right.$ 
can be set to 0$)$ :

$\underset{\mathbf{Q}}{\operatorname{maximize}} \sum_{k=1}^{K} \tilde{r}_{k}\left(\mathbf{Q}_{k} ; \mathbf{Q}^{t}\right)-s^{t, \tau} \sum_{k=1}^{K}\left(P_{0, k}+\rho_{k} \operatorname{tr}\left(\mathbf{Q}_{k}\right)\right)$

subject to $\mathbf{Q}_{k} \succeq \mathbf{0}, \operatorname{tr}\left(\mathbf{Q}_{k}\right) \leq P_{k}, \forall k$.

Since problem (11) is well decoupled across different variables, it can be decomposed into many smaller optimization problems that can be solved in parallel: for all $k=1, \ldots, K$,

$$
\begin{array}{ll}
\underset{\mathbf{Q}}{\operatorname{maximize}} & \tilde{r}_{k}\left(\mathbf{Q}_{k} ; \mathbf{Q}^{t}\right)-s^{t, \tau}\left(P_{0, k}+\rho_{k} \operatorname{tr}\left(\mathbf{Q}_{k}\right)\right. \\
\text { subject to } & \mathbf{Q}_{k} \succeq \mathbf{0}, \operatorname{tr}\left(\mathbf{Q}_{k}\right) \leq P_{k} .
\end{array}
$$

This problem is convex and its optimal point has a closed form expression based on the generalized waterfilling solution [12, Lemma 2]:

$$
\begin{aligned}
\mathbf{Q}_{k}^{\star}\left(s^{t, \tau}\right) & \triangleq \underset{\mathbf{Q}_{k} \succeq \mathbf{0}, \operatorname{tr}\left(\mathbf{Q}_{k}\right) \leq P_{k}}{\arg \max }\left\{\begin{array}{l}
\tilde{r}_{k}\left(\mathbf{Q}_{k} ; \mathbf{Q}^{t}\right) \\
-s^{t, \tau}\left(P_{0, k}+\rho_{k} \operatorname{tr}\left(\mathbf{Q}_{k}\right)\right.
\end{array}\right\} \\
& =\mathbf{V}\left[\mathbf{I}-\boldsymbol{\Sigma}^{-1}\right]^{+} \mathbf{V}^{H},
\end{aligned}
$$

where $[\mathbf{X}]^{+}$denotes the projection of $\mathbf{X}$ onto the cone of positive semidefinite matrices, $(\mathbf{V}, \boldsymbol{\Sigma})$ is the generalized eigenvalue decomposition of $\left(\mathbf{H}_{k k}^{H} \mathbf{R}_{k}\left(\mathbf{Q}_{-k}^{t}\right)^{-1} \mathbf{H}_{k k},\left(s^{t, \tau} \rho_{k}+\right.\right.$ $\left.\left.\rho^{\star}\right) \mathbf{I}-\sum_{j \neq k} \nabla_{\mathbf{Q}_{k}^{*}} r_{j}\left(\mathbf{Q}^{t}\right)\right)$, and $\rho^{\star}$ is the Lagrange multiplier such that $0 \leq \rho^{\star} \perp \operatorname{tr}\left(\mathbf{Q}_{k}^{\star}\left(s^{t, \tau}\right)\right)-P_{k} \leq 0$, which can easily be found by bisection.

After $\left(\mathbf{Q}_{k}^{\star}\left(s^{t, \tau}\right)\right)_{k=1}^{K}$ is obtained, $s^{t, \tau}$ is updated as follows:

$$
s^{t, \tau+1}=\frac{\sum_{k=1}^{K} \tilde{r}_{k}\left(\mathbf{Q}_{k}^{\star}\left(s^{t, \tau}\right) ; \mathbf{Q}^{t}\right)}{\sum_{k=1}^{K}\left(P_{0, k}+\rho_{k} \operatorname{tr}\left(\mathbf{Q}^{\star}\left(s^{t, \tau}\right)\right)\right.} .
$$

It follows from the convergence properties of the Dinkelbach's algorithm (cf. [2]) that

$$
\lim _{\tau \rightarrow \infty} \mathbf{Q}^{\star}\left(s^{t, \tau}\right)=\mathbb{B} \mathbf{Q}^{t}
$$

at a superlinear convergence rate. This iterative procedure (12)(13) is nested under Step 1 of Algorithm 1 as Steps 1.0-1.3.

In the following, we discuss some properties and implementation aspects of the proposed Algorithm 1.

The approximate function in (3) is constructed in the same spirit as $[6,11]$ by keeping as much concavity as possible, namely, $r_{k}\left(\mathbf{Q}_{k}, \mathbf{Q}_{-k}\right)$ in $\mathbf{Q}_{k}$ and $\sum_{j=1}^{K}\left(P_{0, k}+\rho_{k} \operatorname{tr}\left(\mathbf{Q}_{k}\right)\right)$ in $\mathrm{Q}$, and linearizing the nonconcave functions only, namely, $\sum_{j \neq k} r_{j}(\mathbf{Q})$. Besides this, the division operator is also kept. Therefore, the proposed algorithm is of a best-response nature and expected to exhibit a fast convergence behavior, as we shall later illustrate numerically.

In iterative algorithms, the major computational complexity lies in solving the approximate problem in each iteration, or more specifically, the eigenvalue decomposition in (12) with a complexity of $\sum_{k=1}^{K} O\left(M_{T, k}^{3}\right)$. As a result, the complexity is cubic in the number of transmit antennas, and only linear in the number of cells. On the one hand, in the proposed algorithm, the optimal point of the approximate problem has a closedform expression, making the proposed algorithm easy to implement. On the other hand, this also notably accelerates the convergence speed, making the proposed algorithm suitable for real time applications.
The proposed algorithm converges to a stationary point of problem (1) in the sense specified in Theorem 2. Besides, the sequence $\left\{f\left(\mathbf{Q}^{t}\right)\right\}_{t}$ is monotonically increasing. The optimality of the solution to which the algorithm converges is thus always guaranteed.

The proposed algorithm could be implemented by a central unit which has the knowledge of the channel state information of direct-link and cross-link channels, namely, $\left(\mathbf{H}_{k j}\right)_{j, k}$. In practical system, this central unit could be embedded in the Centralized Radio Access Network (CRAN): each BS $k$ measures the direct-link channel $\mathbf{H}_{k k}$ and cross-link channels $\left(\mathbf{H}_{k j}\right)_{j \neq k}$ and send the channel state information $\left(\mathbf{H}_{k j}\right)_{j}$ to the central unit in the CRAN; see the system scenario depicted in Figure 1. Then the central unit invokes Algorithm 1 and informs each BS $k$ the optimal transmit covariance matrix $\mathbf{Q}_{k}$. The incurred latency is mainly due to the signaling exchange between the central unit and the BSs, and the execution of the proposed algorithm.

\section{Simulations}

We consider an urban scenario with a cluster of $K=7$ micro BSs, each serving one UE, as depicted in Figure 1, where the inter-cell distance is $500 \mathrm{~m}$. As mentioned before, this assumption is considered realistic in practical dense urban scenarios, as the number of active users instantaneously connected to a single BS at a specific frequency is low (most of the time only one single user per small cell).

The number of transmit antennas at the BS is $M_{T, k}=4$ and the number of receive antennas at the UE is $M_{R, k}=4$. The power consumption at the zero RF output is $P_{0, k}=16$ $\mathrm{W}$, the power budget normalized by the number of transmit antennas is $36 \mathrm{dBm}$, i.e., $P_{k} / M_{T, k}=36 \mathrm{dBm}$, and the slope of power consumption $\rho$ is 2.6 ; these parameters are mainly adopted from [8]. The simulation results are averaged over 1000 realizations.

For each realization, all $K$ users are randomly located in the multi-cell space where each user falls into the respective hexagonal cell. The following quantities are calculated:

- The optimal transmit covariance matrices $\left(\mathbf{Q}_{k}^{\star}\right)_{k=1}^{K}$, as the output of the proposed Algorithm 1;

- The optimal EE indicator for the entire cluster, as per the objective function in problem (1);

- The benchmark EE, as fair reference for performance comparison with the proposed algorithm, where the transmit covariance matrices are produced by the following two schemes:

- The sum-rate maximizing scheme, i.e., $\left(\mathbf{Q}_{k}\right)_{k=1}^{K}$ maximizes $\sum_{k=1}^{K} r_{k}(\mathbf{Q})$ subject to the constraints: $\mathbf{Q}_{k} \succeq \mathbf{0}, \operatorname{tr}\left(\mathbf{Q}_{k}\right) \leq P_{k}$ for all $k=1, \ldots, K$;

- The uniform transmission scheme, i.e., $\mathbf{Q}_{k}=$ $P_{k} / M_{T x} \cdot \mathbf{I}$;

- EE gain in percentage defined as:

$$
\frac{\text { the optimal EE - the benchmark EE }}{\text { the benchmark EE }} \cdot 100 \% \text {. }
$$

To check if the number of realizations is large enough to average out the randomness in the simulation parameters, 


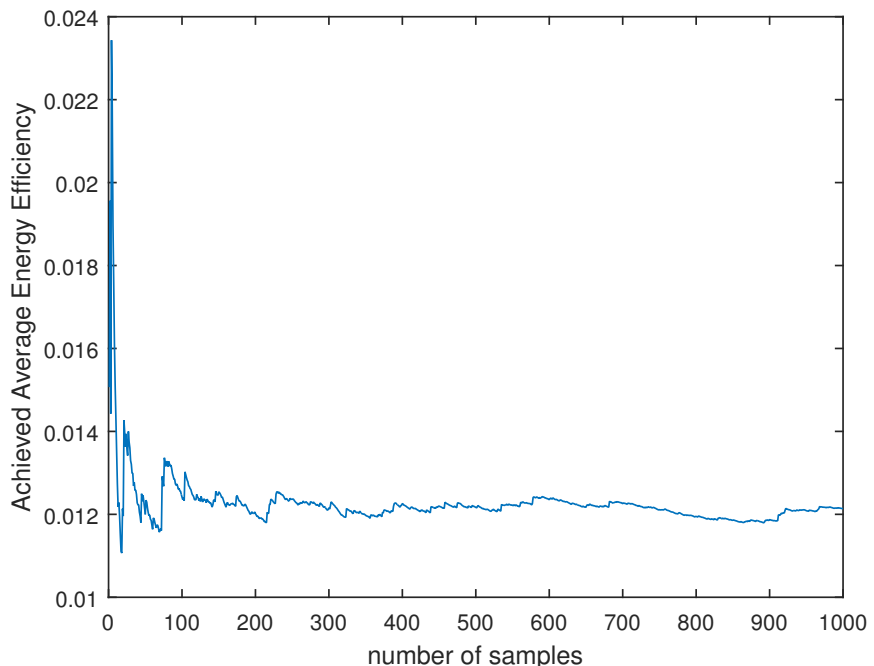

Figure 2. Average EE vs. the number of realizations

we plot in Figure 2 the average EE with the number of realizations. We see that the average EE enters a stable phase after 300 realizations, so the chosen number of realizations, namely 1000, gives us an accurate estimation of the average EE that could be achieved by the proposed scheme.

In Figure 3, we show the convergence speed of the proposed algorithm, and compare the achieved EE by the proposed algorithm and benchmark algorithms. On the one hand, it is easy to see from the black solid line indicating the proposed algorithm that after 2 iterations, the achieved EE is very close to the optimal EE. In practice, the algorithm could be stopped after 2 iterations, which yields a good tradeoff between the achieved EE and the number of iterations. Since the approximate problems solved in each iteration has a closedform solution based on the waterfilling solution, both the computational complexity (linear in the number of cells) and the incurred latency is maintained at a very low level, making the proposed algorithm very suitable in practice. On the other hand, the comparison of the EE achieved by the proposed algorithm and benchmark algorithms (blue dash-dot curve and red dash curve) indicates a notable EE gain. In particular, the EE gain over the uniform transmission is $153.07 \%$ and the $\mathrm{EE}$ gain over the sum-rate optimal transmission scheme is $46.56 \%$.

\section{CONCLUSIONS}

In this paper, we have proposed an efficient optimization algorithm to maximize the EE in interference-limited MIMO systems, based on the recently developed successive pseudoconvex approximation framework. The proposed algorithm not only converges to a stationary point, but also exhibts fast convergence and low complexity, because the structure of the original optimization problem is preserved as much as possible in the approximate problem solved in each iteration, and each approximate problem is natural for parallel computation with a closed-form solution. These advantages are also illustrated by numerical simulations.

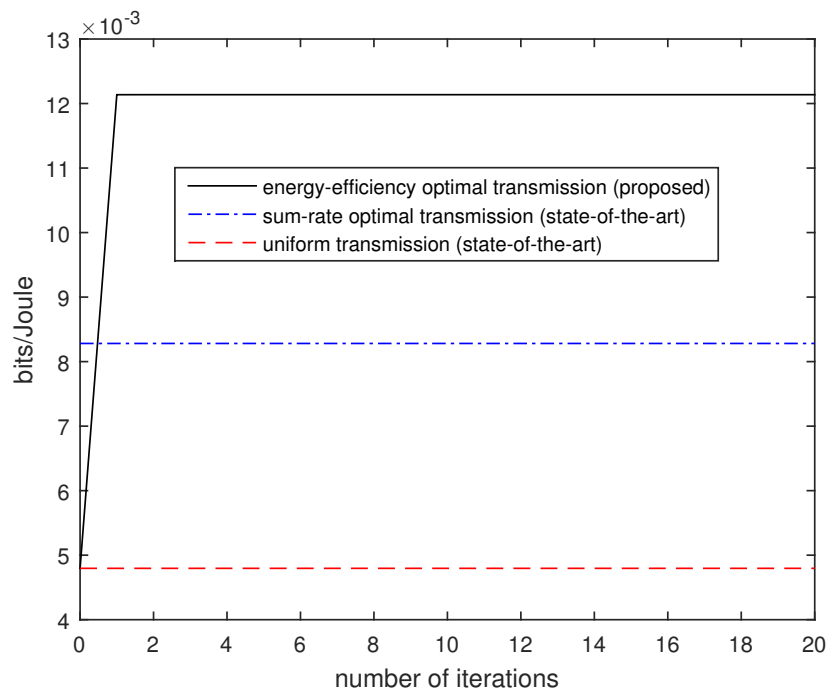

Figure 3. Performance comparison between the proposed scheme and the benchmark schemes

\section{ACKNOWLEDGEMENT}

The work of Y. Yang and D. Sabella was supported by the European Commission in the framework of the H2020-ICT-2014-2 project FANTASTIC-5G (ICT-671660) and Flex5Gware (ICT-671563), respectively.

\section{REFERENCES}

[1] Z.-Q. Luo and S. Zhang, "Dynamic Spectrum Management: Complexity and Duality," IEEE J. Sel. Topics Signal Process., vol. 2, no. 1, pp. 57-73, Feb. 2008.

[2] A. Zappone, L. Sanguinetti, G. Bacci, E. Jorswieck, and M. Debbah, "Energy-Efficient Power Control: A Look at 5G Wireless Technologies," IEEE Trans. Signal Process., vol. 64, no. 7, pp. 1668-1683, April 2016.

[3] O. Tervo, L. N. Tran, and M. Juntti, "Optimal energy-efficient transmit beamforming for multi-user miso downlink," IEEE Transactions on Signal Processing, vol. 63, no. 20, pp. 5574-5588, Oct 2015.

[4] S. Buzzi, C. L. I, T. E. Klein, H. V. Poor, C. Yang, and A. Zappone, "A survey of energy-efficient techniques for $5 \mathrm{~g}$ networks and challenges ahead," IEEE Journal on Selected Areas in Communications, vol. 34, no. 4, pp. 697-709, April 2016.

[5] C. Pan, W. Xu, J. Wang, H. Ren, W. Zhang, N. Huang, and M. Chen, "Pricing-based distributed energy-efficient beamforming for miso interference channels," IEEE Journal on Selected Areas in Communications, vol. 34, no. 4, pp. 710-722, April 2016.

[6] Y. Yang and M. Pesavento, "A unified successive pseudoconvex approximation framework," IEEE Transactions on Signal Processing, vol. 65 , no. 13, pp. 3313 - 3328, Jul. 2017.

[7] A. Zappone, E. Bjornson, L. Sanguinetti, and E. Jorswieck, "Globally optimal energy-efficient power control and receiver design in wireless networks," IEEE Transactions on Signal Processing, vol. 65, no. 11, pp. 2844-2859, Jun. 2017.

[8] "Energy efficiency analysis of the reference systems, areas of improvements and target breakdown," INFSO-ICT-247733 EARTH D2.3. [Online]. Available: https://bscw.ict-earth.eu/pub/bscw. cgi/d71252/EARTH_WP2_D2.3_v2.pdf

[9] D. P. Bertsekas, Nonlinear programming. Athena Scientific, 1999.

[10] O. L. Mangasarian, Nonlinear programming. McGraw-Hill, 1969.

[11] G. Scutari, F. Facchinei, P. Song, D. P. Palomar, and J.-S. Pang, "Decomposition by Partial Linearization: Parallel Optimization of Multi-Agent Systems," IEEE Trans. Signal Process., vol. 62, no. 3, pp. 641-656, Feb. 2014.

[12] Y. Yang, G. Scutari, D. P. Palomar, and M. Pesavento, "A parallel decomposition method for nonconvex stochastic multi-agent optimization problems," IEEE Trans. Signal Process., vol. 64, no. 11, pp. 2949-2964, June 2016. 